\title{
NOTES ON COSTA RICAN PTERICHIS (ORCHIDACEAE) - NEW TAXA AND ADDITIONS TO NATIONAL ORCHID FLORA
}

\author{
Marta KolanowsKa \\ Department of Plant Taxonomy and Nature Conservation, University of Gdańsk \\ ul. Wita Stwosza 59, 80-308 Gdańsk, Poland \\ martakolanowska@wp.pl
}

\begin{abstract}
A new species of the orchid genus Pterichis, $P$. herrerae, and a new variety of $P$. habenarioides, $P$. habenarioides var. costaricensis, are described and illustrated based on Costa Rican material. Information about new Costa Rican records of P. pauciflora and P. parvifolia is provided. An updated key to Costa Rican species of Pterichis is presented.
\end{abstract}

KeY words: Costa Rica, new species, Orchidaceae, Pterichis, taxonomy

Introduction. The Neotropical orchid genus Pterichis was described by John Lindley (1840) based on Peruvian P. galeata. The author considered this species similar to Sarcoglottis C. Presl, but subsequent morphological (Pfitzer 1887) and molecular (eg. Salazar et al. 2003) studies revealed that the two genera are not closely related. Another orchid genus which Lindley found similar to his newly established Pterichis in the gynostemium structure was Cryptostylis R. Br., but this genus was classified by Szlachetko \& Rutkowski (2000) in different subfamilies based i.a. on their anther form and position. Molecular data (Salazar et al. 2009) indicates that Pterichis is sister to other Cranichidinae (e.g., Cranichis Sw., Ponthieva R. Br. and Baskervilla Lindl.).

Morphologically representatives of Pterichis are easily distinguished from other cranichid orchids based on their floral characters. The abbreviate, massive gynostemium is observed also in Cranichis, Fuertesiella Schltr., Pseudocentrum Lindl. and Ponthieva. From the two latter genera Pterichis differs in the petals and lip being free from the column part and from other two by the scape developing separately from leaves.

Plants of Pterichis produce tuberous, clustered roots. The non-resupinate flowers are arranged into a loosely to subdensely flowered raceme (rarely congested). The tepals of Pterichis are subsimilar, with the petals narrower than the dorsal sepal and often adnate to it. The sessile lip is concave, usually with a pubescent disc and ornamented with swollen cells or knob-like projections along the margins. The gynostemium is short and erect, and the motile anther is much longer than the rest of the column. Four oblong-ovoid pollinia are produced. The rostellum is narrow, delicate, filiform and the hamulus is finger-like (Szlachetko \& Rutkowski 2000).

The plants of Pterichis grow terrestrially and they are usually found in grassy slopes or shrubby paramo above $2800 \mathrm{~m}$ of elevation. Most of the currently recognized 25 species are distributed along the Andes; however, some species occur in Jamaica, Panama and Costa Rica (Cribb 2003, Dueñas Gómez \& Fernández Alonso 2009).

Dressler (2003) reported two species of Pterichis from Costa Rica: P. habenarioides (F. Lehm. \& Kraenzl.) Schltr. and $P$. galeata Lindl. considering $P$. costaricensis Ames \& C. Schweinf. and P. leo L.D. Gómez \& Gómez-Laur. as their respective synonyms. The Costa Rican populations are found in the elevations above $2250 \mathrm{~m}$, often in wet montane forest and paramo.

During recent studies of herbarium specimens a new species of Pterichis was found and is described here. Moreover, an unusual form of $P$. habenarioides recognized in Costa Rican material is proposed here as a new variety. Two species of Pterichis, P. pauciflora Schltr. and $P$. parvifolia (Lindl.) Schltr. are reported here for Costa Rica for the first time.

Material and Methods. Dried herbarium specimens of Pterichis, over 180 in total, deposited or borrowed from AMES, COL, CUVC, F, FLAS, FMB, HUA, K, 

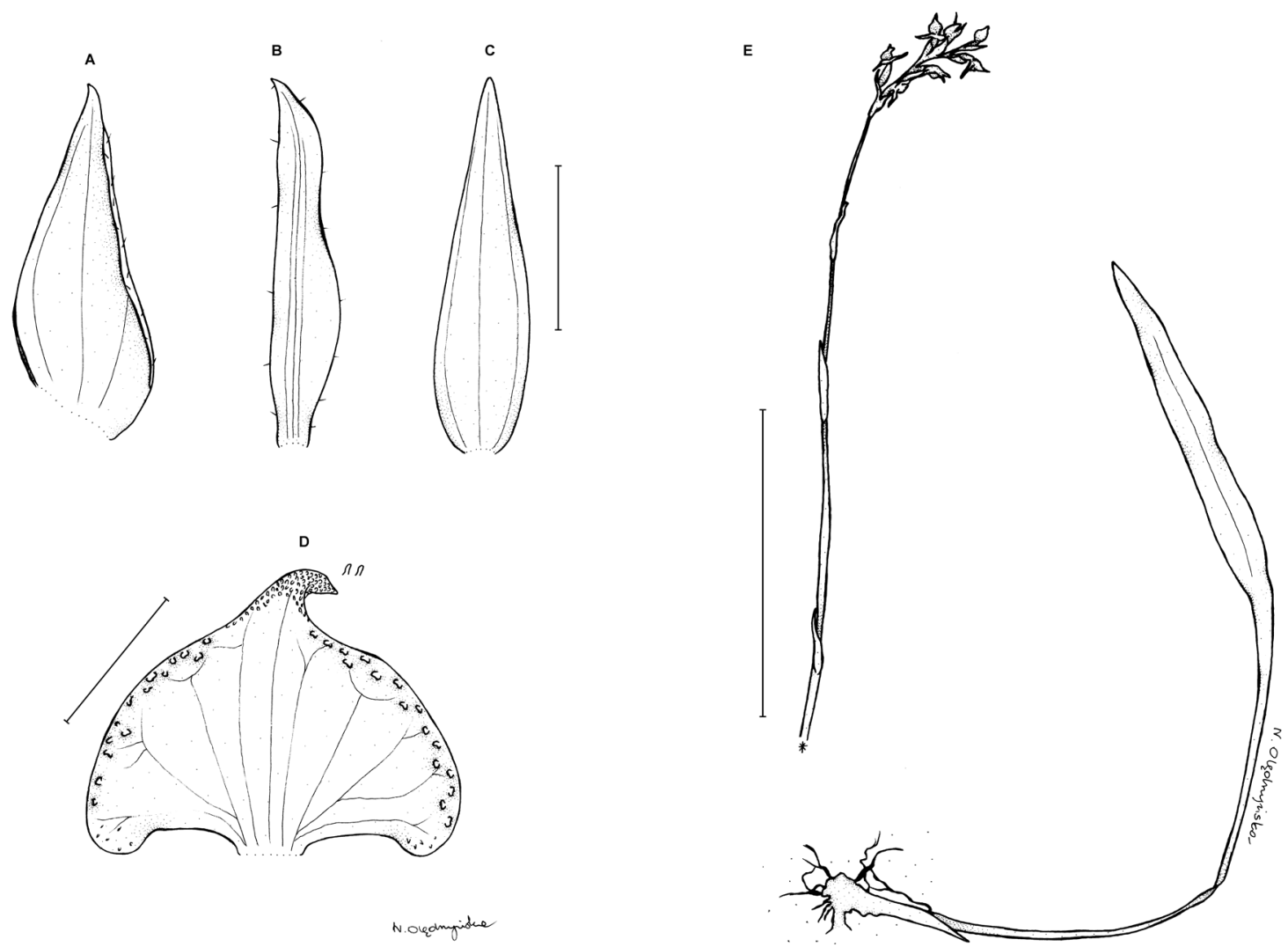

Figure 1. Pterichis herrerae A. Lateral sepal. B. Petal. C. Dorsal sepal. D. Lip. Scale bars $=3 \mathrm{~mm}$. E. Scape, spike and leaf. Scale bar $=3 \mathrm{~cm}$. Drawn by N. Olędrzyńska from the holotype.

MO, NY, P, PSO, SEL, VALLE and W were examined. Every studied specimen was photographed and the data from the labels were recorded. The presence and shape of the leaf as well as the length and the surface of the scape were studied. The examination of the vegetative structures included the form and number of the tubular sheaths enveloping the scape and the form of the floral bracts and ovaries. From each specimens 3-5 flowers were examined. The perianth parts were studied after rehydrating flowers in boiling water.

\section{NEW TAXA}

Pterichis herrerae Kolan., sp. nov.

TYPE: Costa Rica. Puntarenas. Cantón de Buenos Aires, Ujarrás, cumbre y ladera Atlántica de Cerro

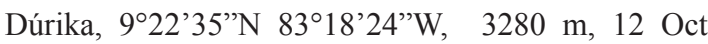
1989, G. Herrera 3665 (holotype, F!; isotypes, F!, MO, SEL). Fig. 1-2.
Diagnosis. This species resembles $P$. habenarioides and $P$. triloba (Lindl.) Schltr., but it is distinguished by the cordate lip base, obliquely ovate, rounded lip lateral lobes and lip margins ornamented with prominent, knob-like projections.

Plant up to $32 \mathrm{~cm}$ tall. Leaf basal, long-petiolate; blade $9.5 \mathrm{~cm}$ long, linear-lanceolate, acute; petiole 12 $\mathrm{cm}$ long. Scape up to $27 \mathrm{~cm}$ long, with 4 glabrous, tubular sheaths, pubescent in the upper part. Spike $2.7-5.0 \mathrm{~cm}$ long, pubescent, subdensely 4-10-flowered. Flowers green with yellowish-green lip. Floral bracts up to $8 \mathrm{~mm}$ long, $3 \mathrm{~mm}$ wide, pubescent, narrowly ovate, subobtuse. Ovary $9 \mathrm{~mm}$ long, pubescent. Dorsal sepal $7.0 \times 1.5 \mathrm{~mm}$, externally sparsely ciliate, lanceolate, obtuse, 3-veined. Petals $6.8 \times 1.2 \mathrm{~mm}$, adnate to dorsal sepal, sparsely ciliate along margins, linear-lanceolate, unguiculate at the base and constricted in the upper third, acute, 3-veined. Lateral sepals $6.5 \times 2.5 \mathrm{~mm}$, 

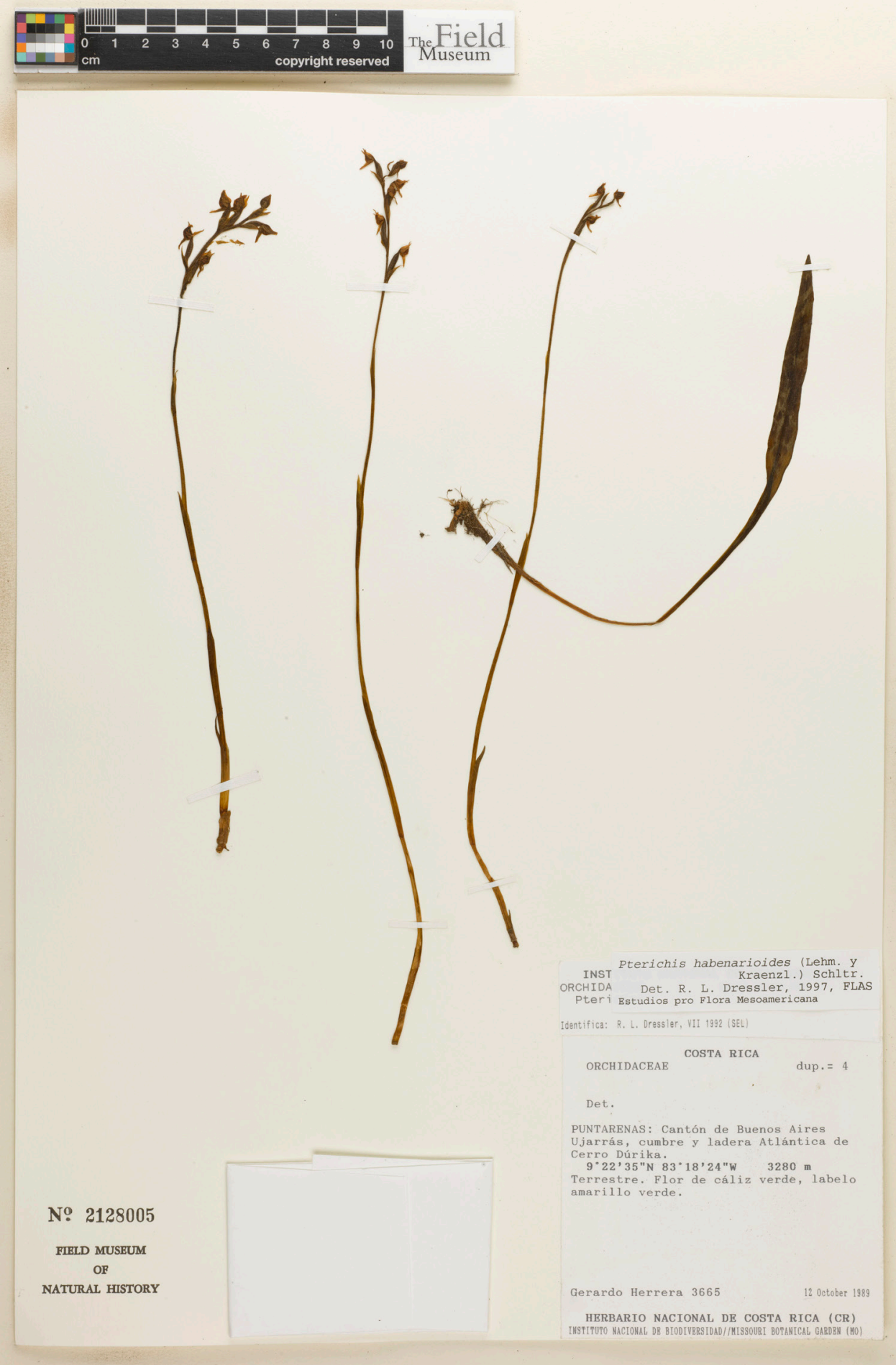

FIgure 2. The holotype of Pterichis herrerae. Courtesy of the Field Museum of Natural History. 
I

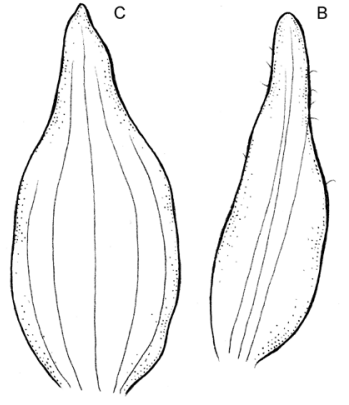

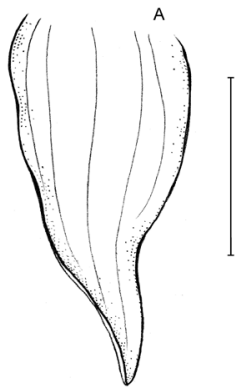
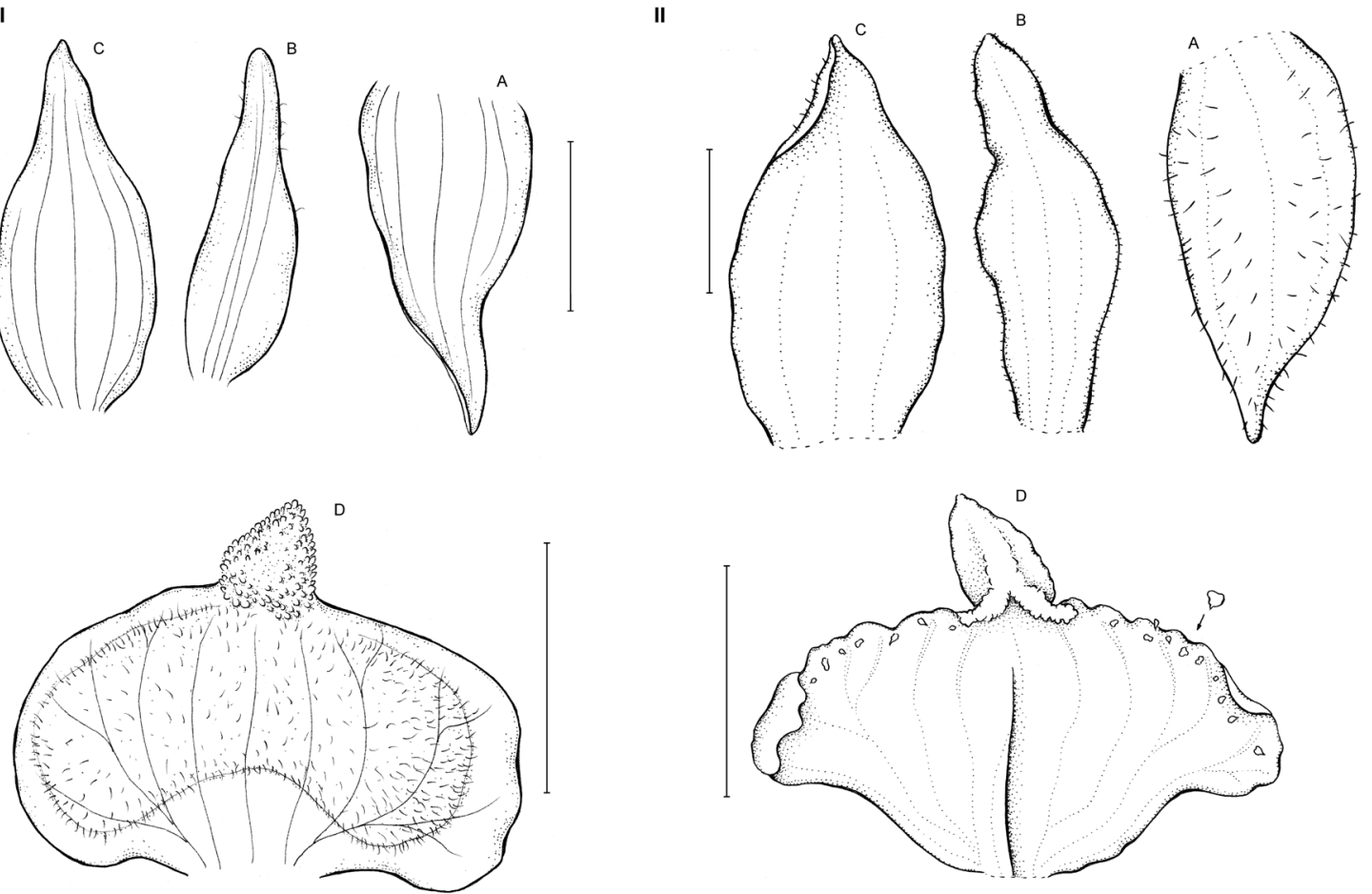

Figure 3. Perianth segments of Pterichis habenarioides (I, Pedraza \& al. 247, COL) and P. triloba (II, Jameson s.n., W). A.

Dorsal sepal. B. Petal. C. Lateral sepal. D. Lip. Scale bars $=3 \mathrm{~mm}$. Drawn by A. Król and S. Nowak.

externally sparsely ciliate, obliquely ovate, subacute, 2-veined. Lip about $5 \times 7 \mathrm{~mm}, 3$-lobed, cordate at the base; middle lobe about $1 / 4$ of the lip length, reflexed, triangular-ovate, acute, densely glandular-pubescent; lateral lobes obliquely triangular-ovate, rounded; disc 5-veined, veins branching, margins ornamented with knob-like projections and swollen cells in the basal part. Gynostemium $2.5 \mathrm{~mm}$ long. Fruit not seen.

Etymology. Dedicated to collector of the type specimen.

Distribution AND ECOlOGy. So far this species is known exclusively from Costa Rican province of Puntarenas, where it was found growing terrestrially at the altitude of over $3200 \mathrm{~m}$. Flowering occurs at least in October.

TAXONOMIC NOTES. This species resembles $P$. habenarioides (F.Lehm. \& Kraenzl.) Schltr. and $P$. triloba (Lindl.) Schltr. (Fig. 3). From the latter species $P$. herrerae is easily distinguished by the cordate lip base (vs. truncate in $P$. triloba), the 5-veined lip disc (vs. 7-veined) and presence of numerous (over 30 in total) knob-like projections along the lip margins (vs.
8-10 projections on each side). The floral bracts of $P$. habenarioides are glabrous, its dorsal sepal is ovatelanceolate (vs. lanceolate in $P$. herrerae), the petals are obovate-lanceolate, not unguiculate (vs. linear-lanceolate above, unguiculate basally), and the lip is transversely triangular-elliptic in outline (vs. cordate in $P$. herrerae). Another similar species is the South American $P$. diuris Rchb. f., which has petals free from dorsal sepal, the lip base is truncate and disc lacks any knob-like projections.

Pterichsis habenarioides var. costaricensis Kolan., var. nov.

Diagnosis: Distinguished from $P$. habenarioides var. habenarioides by the glabrous dorsal sepal and petals, the sparsely ciliate, 2-veined lateral sepals, and the lateral lobes of the lip form auricles.

TYPE: Costa Rica. Limon. Cordillera de Talamanca, Atlantic slope, Kámuk massif, páramo north-east of the main Kámuk peak, 9¹6’30”N 8301'00"W, 3000-3300 m, 17-19 Sep 1984, G. Davidse \& G. Herrera 29296 (holotype, F!; isotype: CR). Fig. 4-5. 

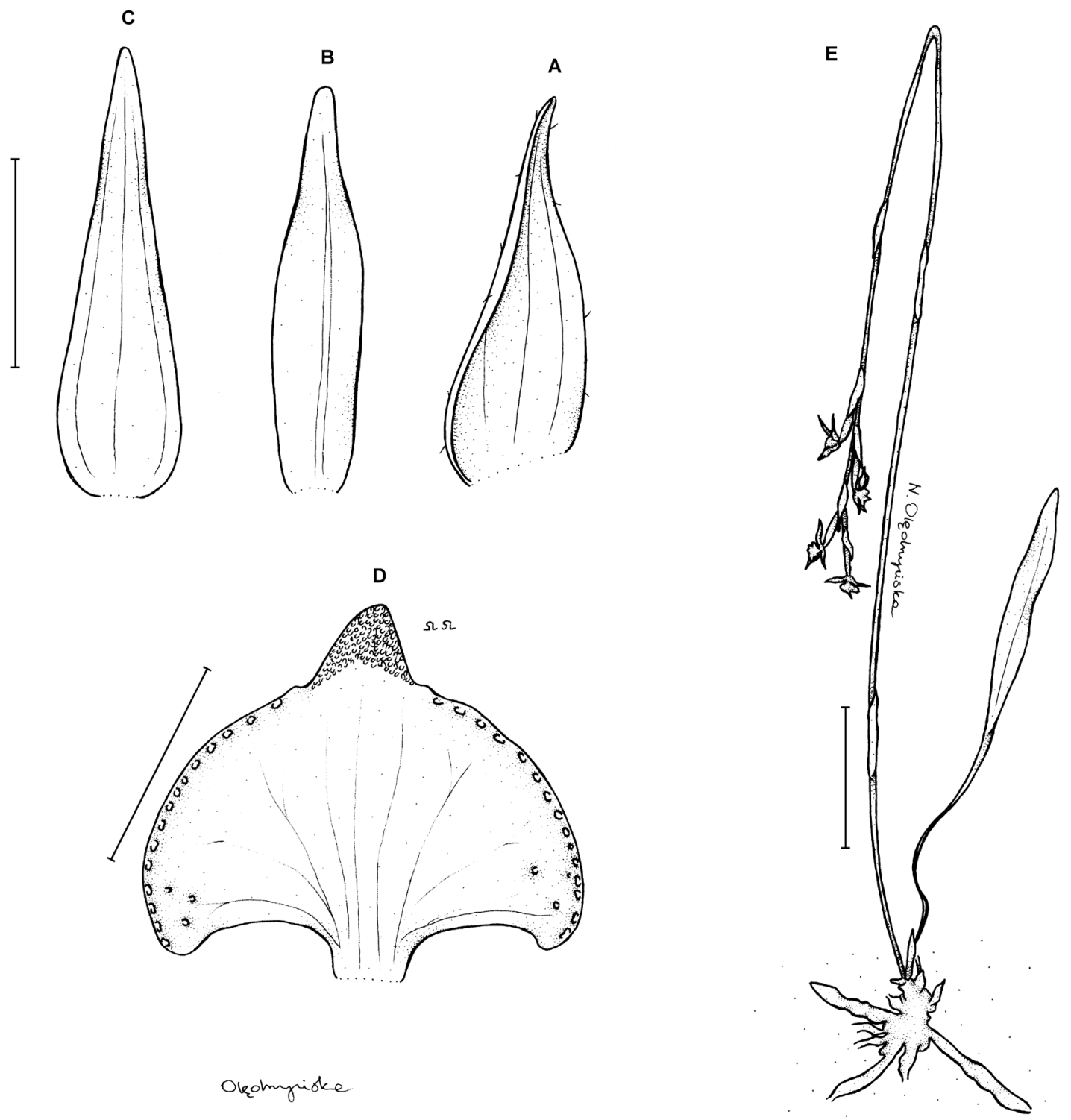

Figure 4. Pterichsis habenarioides var. costaricensis A. Lateral sepal. B. Petal. C. Dorsal sepal. D. Lip, flattened. E. Habit. Scale bar $=3 \mathrm{~cm}$. Drawn by N. Olędrzyńska from the holotype.

Plants up to $40 \mathrm{~cm}$ tall. Leaf basal, petiolate; blade lanceolate, obtuse, 2-veined. Lateral sepals $5.5 \times$ 5.5-6.0 cm long, linear-lanceolate, acute; petiole 4.5- $1.8 \mathrm{~mm}$, ovate, obtuse, sparsely ciliate, 2-veined. $6.5 \mathrm{~cm}$ long. Scape up to $36 \mathrm{~cm}$ long, scape with 3-4 Lip $6 \mathrm{~mm}$ long and about the same wide, indistinctly tubular sheaths. Flower greenish-yellow, lip yellow 3-lobed; middle lobe broadly ovate, short, densely with green or brown veins. Floral bracts up to $13 \mathrm{~mm}$ glandular-pubescent, obtuse, reflexed; lateral lobes long, $4 \mathrm{~mm}$ wide, pubescent. Ovary up to $13 \mathrm{~mm}$ long, pubescent. Dorsal sepal $6.5 \mathrm{~mm}$ long, $1.5 \mathrm{~mm}$ wide, glabrous, lanceolate, obtuse, 3-veined. Petals $5.9 \times$ $1.2 \mathrm{~mm}$, adnate to dorsal sepal, glabrous, ligulateobliquely ovate, rounded; disc 5-veined, lateral veins branching, ornamented with numerous swollen cells along the margins. Gynostemium $2.5 \mathrm{~mm}$ long. Fruit not seen. 

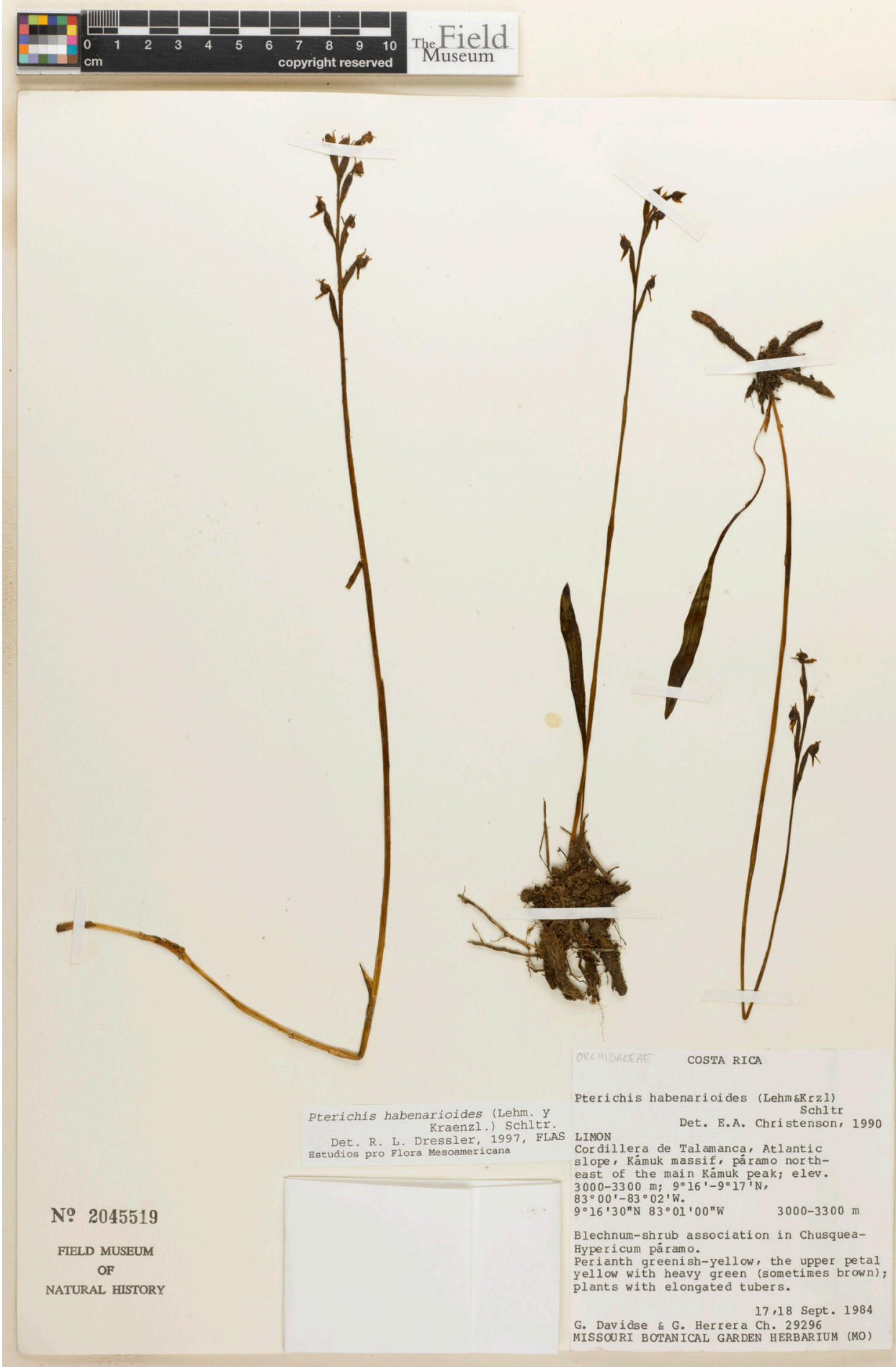

FiguRE 5. The holotype of Pterichis habenarioides var. costaricensis. Courtesy of the Field Museum of Natural History. LANKESTERIANA 14(2), August 2014. (C) Universidad de Costa Rica, 2014. 
Etymology: In reference to the origin of the type specimen.

Distribution AND ECOLOGY. So far this variety is known exclusively from Cordillera de Talamanca. The population was found growing in Blechnumshrub association in Chusquea-Hypericum paramo. Flowering occurs in September.

EXAMINED SPECIMENS OF $P$. HABENARIOIDES VAR. HABENARIOIDES: Colombia. Boyacá. Mpio. de Chinavita. Cerro Mamapacha. Vereda Mundo Nuevo, Laguna La Jarilla, paramo, 3300 m, 26 Jul 2001, H. Duenas, F. Cortes, J. Farfan, E.C. Buitrago, M.F. Rodriguez \& J. Puentes 3107 (COL!), Cauca. Mpio. de Purace. Parque Nacional Natural de Purace. Cercanias de la Laguna San Rafael, 3300 m, 6 Oct 1984, G. Lozano, O. Rangel, L.F. de Turbay, A. Sanabria \& N. Espejo 4654 (COL!), Macizo Colombiano. Paramo de Las Papas. Colinas al SE de la Laguna La Magdalena, vertiente del rio Magdalena, 3350-3450 m, 12 Sep 1958, Idrobo, Pinto \& Bischler 3249 (COL!), Macizo Colombiano. Paramo Las Papas. Filo entre el Boqueron y el cerro de Penas Blancas, 3330 m, 14 Sep 1958, Idrobo, Pinto 7 Bischler 3321 (COL!), Macizo Colombiano. Valle del Cauca, cerca de su nacimiento, 3000 m, 19 Sep 1958, Idrobo, Pinto \& Bischler 3460 (COL!), Macizo Colombiano. Valle de Las Papas, alrededores de Valencia, Los Andes. Alt. 3150 m. Wet cienga between scrub patches, an extension of Station 4, approx. $3 \mathrm{~km}$ from casa of Hacienda Los Andes, 1 Oct 1958, Barclay \& P. Juajibioy 5892 (COL!), Paramo Guanacas. Lehmann 6419 (K!), Cundinamarca. D.C., localidad 20, Parque Nacional Natural Sumapaz, vereda Santa Rosa, arriba de la laguna Casa Blanca, 3500 m, 7 Aug 1998, P. Pedraza, P. Franco, D. Stancik \& A. Neira 247 p.p. (COL!), Bogotá. Paramo de Cruz Verde, 3200 m, 6 Sep 1977, G. Morales \& Flenley 30 (COL!), San Miguel. Schneider 673 (COL!), Huila. Mpio. de Gigante. Vered Ventanas, Cerro de Miraflores, subida desde el Campamento 1, en bosque andino de Winmannia, Brunellia, Clusia hasta el subparamo (3050 m), con Dipostephium, Brachyotum, Gynoxis, Weinmannia, 12-16 Aug 1997, J.L. Fernandez Alonso, E. Linares, P. Balcazar, R. Vasquez, J. Velez \& G. Salazar 14851 (COL!), Meta. Macizo de Sumapaz. Alrededores de la Laguna La Guitarra, alt. 3380-3420 m, 4 Jul 1981, S. Diaz P. 2404 (COL!), Nariño/Putumayo. Mpio. de
Santiago Vereda. Paramo del Bordoncillo. $1^{\circ} 11^{\prime} \mathrm{N}$, 7706'W, 3200-3400 m, 17 Apr 1993, A. Munoz \& D. Ramirez 414 (COL), Quindio. Pijao. Paramo del Chili, km 28, paramo, 3600 m, 17 Sep 1998, Gil-Correa 115 \& N. Ocampo, M. Piepenbring, C. Velez (COL!), Sine loc. Lehmann 6419 (K!).

NEW RECORDS

Pterichis pauciflora Schltr., Repert. Spec. Nov. Regni Veg., Beih. 8: 41. 1921.

TYPE(Garay 1978): Ecuador. Loja, in Andibus orientalibus, 3000-3400 m, Lehmann 7111 (holotype: $\mathrm{B} \dagger$, lectotype, $\mathrm{K}$ !).

Plant $14-62 \mathrm{~cm}$ tall, erect. Leaf basal, petiolate; petiole 2-3 cm long; blade about $5 \times 1-2 \mathrm{~cm}$, linear to oblong-lanceolate, acute. Scape up to $58 \mathrm{~cm}$ tall, erect, minutely puberulent in the upper part, with 3-6 tubular, pubescent sheaths. Spike 2-4 cm long, pubescent, loosely to subdensely 5-10-flowered. Flowers yellowish-orange to yellowish-brown according to the herbarium label. Floral bracts up to $8 \mathrm{~mm}$ long, ovate-lanceolate, glabrous. Pedicellate ovary 9-11 $\mathrm{mm}$ long, densely glandular. Dorsal sepal $6.0-6.8 \times$ 1.7-2.2 mm, ovate-lanceolate to ovate, obtuse, almost glabrous, 3-veined. Petals $6.0-6.5 \times 1.0-1.2 \mathrm{~mm}$, adnate to dorsal sepal, obliquely linear-lanceolate, acuminate, margin sparsely ciliate, 1-veined. Lateral sepals $5.5-6.5 \times 1.5-2 \mathrm{~mm}$, obliquely ovate-lanceolate to ovate, concave, acuminate, glabrous or sparsely ciliate externally, 3-4-veined. Lip 5,0 × 4.5-7.0 mm, indistinctly 3-lobed; basal part triangular-elliptic; apical lobe triangular, obtuse, reflexed, glandulosepapillose; disc 7-veined, margin ornamented with swollen cells. Gynostemium $2.3 \mathrm{~mm}$ long. Fruit not seen.

Representative specimens: Costa Rica. Puntarenas. Cantón de Coto Brus. P.N. La Amistad, Cuenca Térraba-Sierpe, Cerro Echandi, 901'57'N 8249'19''W, 3000-3150 m, 13 Aug 1997, Gamboa \& al. 1718 (FLAS!); Cartago. Carretera Panamericana Sur, 2700 m, 17 Jul 1963, A. Jimenez 849 (F!, Fig. 6).

Distribution and eCology. Until now this species was reported from Ecuador and Colombia. In Costa Rica it was found growing terrestrially in paramo, forest edges and vegetation with Sphagnum at the altitude 

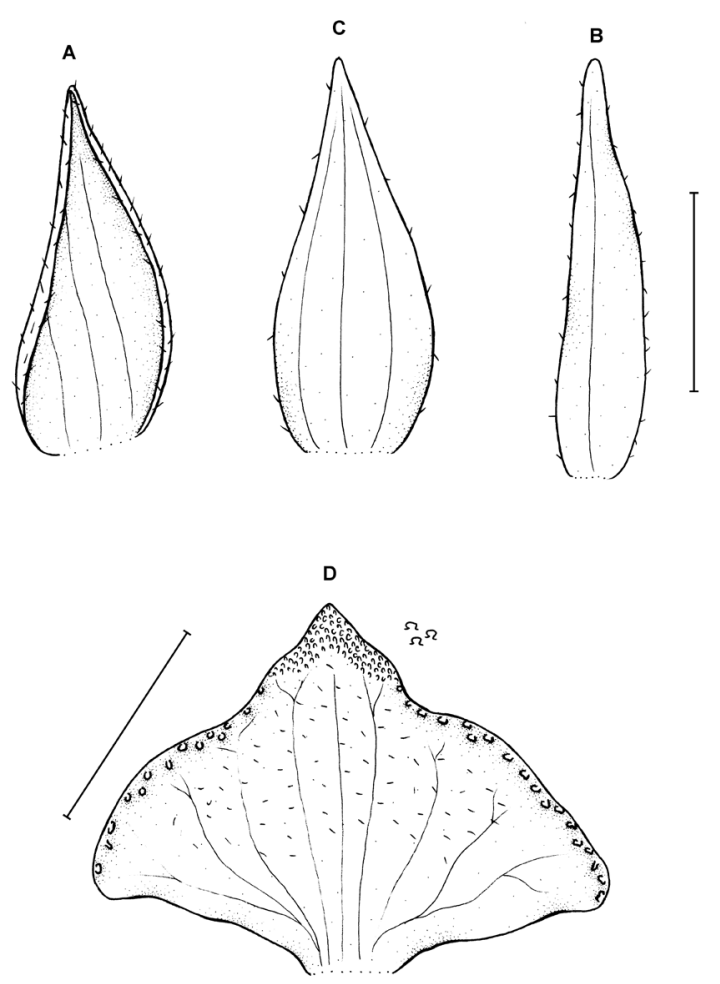

$$
\text { Okolngrioke }
$$

Figure 6. Pterichis pauciflora A. Lateral sepal. B. Petal. C. Dorsal sepal. D. Lip. Scale bars $=3 \mathrm{~mm}$. Drawn by N. Olędrzyńska from Jimenez 849 (F).

above $2700 \mathrm{~m}$. Flowering occurs in July and August. Other SPECIMENS EXAMINED: Colombia. Boyacá. Mpio. Arcabuco. Santuario de Flora y Fauna de Igauque. Camino a la Laguna. Paramo dominado por Espeletia, Calamagrostis, Hypericum, Lupinus, Monnina, Cavendishia, Castilleja, 3600 m, 24 Sep 2002, H. Duenas, F. Cortes \& N. Aranguren 3130 (COL!), Mpio. Villa de Leyva. Parque Nacional Natural santuario de Iguaque, 3700 m, 20 Jul 1979, M. Melampy 207 (COL!), Mpio. Pesca. Paramo de la Cortadera. Vereda La Pena, 3600 m, 21 Aug 1982, M. Bejarano B. 245 (COL!), Cundinamarca. D.C., localidad 20, Parque Nacional Natural Sumapaz, vereda Santa Rosa, arriba de la laguna Casa Blanca, 3500 m, 7 Aug 1998, P. Pedraza, P. Franco, D. Stancik \& A. Neira 247 p.p. (COL!), Bogotá. Paramo de Monserrate, 3000 m, 26 Jul 1943, Schneider 243 (COL!), Carretera Paramo de Palacio a Rio Chuza, cabeceras Valle Chocolatel.
Vertiente pedregoso con Calamagrostis effusa y Oreobolus obtusangulus, 3700 m, 9 Sep 1972, A.M. Cleef 5435 (COL!), Paramo entre Cogua y San Cayetano. Laguna Verde y alrededores Paramo seco con Calamagrostis effusa, $2 \mathrm{~km}$ al NW de la Laguna, 3600 m, 19 Aug 1972, A.M. Cleef, M.T. Murillo \& van der Hammen 5164 (COL!), Meta. Macizo de Sumapaz. Alrededores de la Laguna El Sorbedero, 3550 m, 4 Jul 1981, S. Diaz P., A.M. Cleef, van der Hammen, O. Rangel \& S. Salamanca 2472 (COL!), Upland E of Sesquile, 3300 m, 5 Jul 1947, Haught 5929 (COL!). Ecuador. Loja. Lehmann 7111 (K!).

Pterichis parvifolia (Lindl.) Schltr., Bot. Jahrb. Syst. 45: 389. 1911. 三 Acraea parvifolia Lindl., Pl. Hartw.: 155. 1845.

TYPE: Ecuador. Loja. Hartweg 50 (K-Lindl.!).

Plant about $37 \mathrm{~cm}$ tall. Leaf basal, petiolate; petiole $6.5-9 \mathrm{~cm}$ long, canaliculated; blade up to 11.0 $\times 0.6-1.0 \mathrm{~cm}$, linear-lanceolate, subacute. Scape about $25 \mathrm{~cm}$ tall, pubescent, with 5 sheaths decreasing in size distally along the scape, pubescent in upper half. Spike 8-9 cm long, pubescent, loosely several-flowered. Flowers greenish with yellow lip with brownish veins. Floral bracts up to $9 \mathrm{~mm}$ long, glabrous, ovatelanceolate, acute. Pedicellate ovary up to $13 \mathrm{~mm}$ long, pubescent. Dorsal sepal $7 \times 2 \mathrm{~mm}$, ovate-lanceolate, subacuminate, obtuse, glabrous, 3-veined. Petals 7.0 $\times 1.5 \mathrm{~mm}$, ciliate along margins, unguiculate in lower fifth, obliquely oblong-lanceolate, subacute, 2-veined. Lateral sepals $5.5 \times 2.5 \mathrm{~mm}$, glabrous, obliquely ovate, subacuminate, 2-veined. Lip about 5-6 $\times 7 \mathrm{~mm}$, 3-lobed, base truncate; middle lobe reflexed, densely glandular-pubescent, ovate-triangular, subobtuse; lateral lobes obliquely ovate; disc 5-veined, lateral lobes branching, margin ornamented with a row of prominent papillae. Gynostemium $2 \mathrm{~mm}$ long. Fruit not seen.

Representative specimen: Costa Rica. San Jose and Cartago. Below the highest point of Interamerican

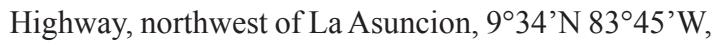
3000-3200 m, 27 Oct 1975, W. Burger \& R. Baker 9505 (F!, Fig. 7).

Distribution And ECOLOgy. Until now this species was reported from Peru, Ecuador and Colombia. In 


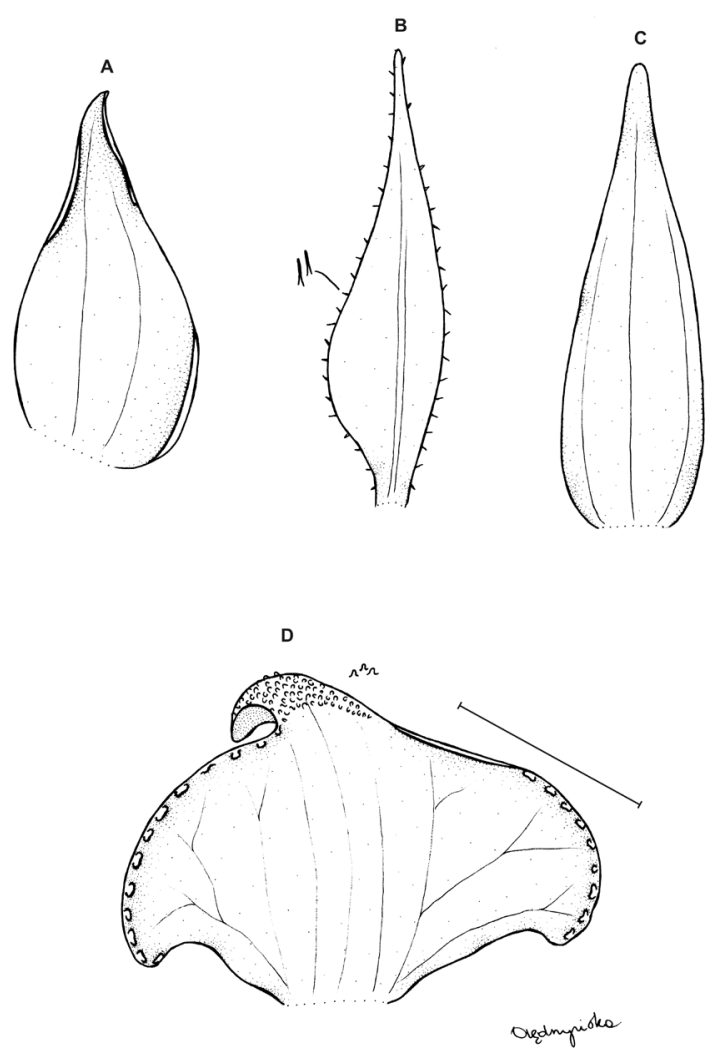

Figure 7. Pterichis parvifolia A. Lateral sepal. B. Petal. C.

Dorsal sepal. D. Lip. Scale bars $=3 \mathrm{~mm}$. Drawn by N. Olędrzyńska from Burger \& Baker 9505 (F).

Costa Rica it was found in steep wet grassy slope at an altitude of about 3000-3200 m. Flowering occurs at least in October.

OTHeR SPECIMENS EXAMINED: Colombia. Nariño. Mpio. Guachucal-Cumbal. Paramo de Infernillo. The ridge in direction to Vulcan Cumbal. Grassy paramo with Calamagrostis effusa, Cortadera, Diplostephium, Espeletia, Puya. Flowers yellowish, 3700 m, 5-9 Mar 1999, Stancik 2687 (COL!), Mpio. MallamaPiedrahancha. Vereda Pueblo Viejo. Paramo del Infernillo. Reserve Natural Pueblo Viejo. Km 97 via Pasto-Tumaco, desvio a la izquierda viajando hacia Tumaco y ascenda de $2 \mathrm{~km}$ por la carretera via Pueblo Viejo Coleccion entre la Cabana principal de la reserve y el sitio "La Lagunetas", $1^{\circ} 03^{\prime} 02.9^{\prime \prime}-1^{\circ} 01{ }^{\prime} 16.2^{\prime \prime} \mathrm{N}$,

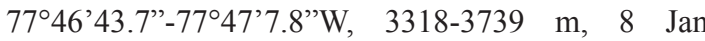
2009, L.M. Caballero, O. Rivera Diaz, G. Silva, C. Leguizamo, M. Ayala, J. Gil \& M. Rodriguez LMC3 (COL!). Ecuador. Loja. Hartweg 50 (K-Lindl.!).
Key to Costa Rican species of Pterichis

1. Petals free from dorsal sepal P. galeata

1a. Petals adnate to dorsal sepal 2

2. Lateral lobes of the lip not forming auricles P. habenarioides var. habenarioides

2a. Lateral lobes of the lip forming auricles 3

3. Petals glabrous

P. habenarioides var. costaricensis

3a. Petals ciliate on margins 4

4. Petals sessile, 1-veined ...... P. pauciflora 4a. Petals unguiculate, 2-veined …….......... 5

5. Petals constricted in the upper third, sepals externally ciliate $P$. herrerae

5a. Petals not contricted in the upper part, sepals glabrous ........ P. parvifolia

Acknowledgments. Anna Król, Natalia Olędrzyńska and Sławomir Nowak are thanked for preparing illustrations. The Curators and staff of the cited herbaria are thanked for providing access to their collections. I am grateful to Professor Dariusz L. Szlachetko for his valuable comments on the manuscript. The research described here was financed by the Faculty of Biology, University of Gdańsk (538-L150-B065-13).

LiteratuRE CITED

Cribb, P. 2003. Pterichis. Pp. 53-56. in: A. M. Pridgeon, P. J. Cribb, M. C. Chase \& F. N. Rasmussen (eds.), Genera Orchidacearum. New York, Oxford, Oxford University Press.

Dressler, R. L. 2003. Orchidaceae. Pp. 1-595. in: B. E. Hammel, M. H. Grayum, C. Herrera \& N. Zamora (eds.), Manual de Plantas de Costa Rica, vol III: Monocotiledóneas (Orchidaceae-Zingiberaceae). Monogr. Syst. Bot. Missouri Bot. Gard. 93: 1-884.

Dueñas Gómez, H.C., Fernández Alonso, J.L. 2009. Sinopsis de la Subfamilia Spiranthoideae (Orchidaceae) en Colombia, parte II. Rev. Acad. Colomb. Ci. Exact. 33( 127): 157-181.

Garay, L. A. 1978. 225(1) Orchidaceae, Cypripedioideae, Orchidoideae, Neottioideae. Pp. 1-305. in: G. Harling \& B. Sparre (eds.), Flora of Ecuador 9. University of Goteborg, Stockholm, Sweden.

Lindley, J. 1840. The Genera and Species of Orchidaceous Plants. Part 7(1): 441-528.

Pfitzer, E. H. H. 1887. Entwurf einer natürlichen Anordnung der Orchideen. Carl Winter's Universitätsbuchhandlung, Heidelberg, Germany.

Salazar, G. A., M. W. Chase, M. A. Soto \& M. Ingrouille. 2003. Phylogenetics of Cranichideae with emphasis on 
Spiranthinae (Orchidaceae, Orchidoideae): evidence from plastid and nuclear DNA sequences. Am. J. Bot. 90: 777-795.

Salazar, G. A., L. I. Cabrera, S. Madriñán \& M. W. Chase. 2009. Phylogenetic relationships of Cranichidinae and
Prescottiinae (Orchidaceae, Cranichideae) inferred from plastid and nuclear DNA sequences. Ann. Bot. 104(3): 403-416.

Szlachetko D. L. \& P. Rutkowski. 2000. Gynostemia Orchidalium 1. Acta Bot. Fenn. 169: 1-380. 